\title{
Ligand-Binding Assays: Development, Validation and Implementation in the Drug Development Arena
}

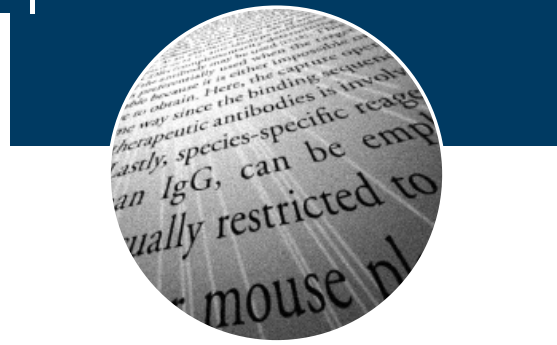

\author{
"Ligand-Binding Assays, written by recognized and respected industry experts, covers a wide range of topics that \\ provide valuable information to someone who is new to the field..."
}

The past decade has seen significant changes in ligand-binding assays (LBAs) largely due to advances in technology - both in reagent technologies, such as monoclonal antibody generation, as well as with detection and instrumentation technologies. These advances coupled with the growing number of therapeutic macromolecules have stirred the scientific and regulatory communities to come together to define the requirements for bioanalytical methods for macromolecules. The requirements for and the challenges of LBAs have been discussed and debated in various forums. At this juncture in the history of LBAs, there arises the need for a comprehensive text that can serve as a reference guide for the entire bioanalytical community. Ligand-Binding Assays, written by recognized and respected industry experts, covers a wide range of topics that provide valuable information to someone who is new to the field, as well as in-depth discussion of complex issues for the advanced bioanalytical scientist.

Drug development has seen an upsurge in the number of therapeutic macromolecules or 'biologics' over the past few years. The bioanalysis of macromolecules in complex matrices cannot be performed by MS, which is the dominant technology in the bioanalysis of low-molecular weight xenobiotics. LBAs are the preferred bioanalytical methods for biologics in support of pharmacokinetic and toxicokinetic studies as well as for immunogenicity testing and for several biomarker assays. Ligand-binding methods are typically immunoassays that utilize macromolecular interactions between the analyte of interest and another protein, receptor, ligand or antibody for the detection and quantitation of the analyte. Due to the differences between small and large molecules, assay-performance parameters that are of concern may be different for LBAs as compared with MS methods for xenobiotics. In its most common format, an LBA requires a capture molecule that captures the analyte or drug of interest and a detector molecule that detects the captured analyte. There are many variations on this central theme in terms of assay formats, capture and detection molecules as well as detection technologies. The most common LBA is the ELISA in which the complex is captured on a solid surface and the detector molecule is linked to an enzyme. Addition of a substrate results in the enzymatic conversion to a colored product that is detected by measurement of absorbance. Over the past few decades several new detection technologies have emerged that utilize varying detection systems such as fluorescence, time-resolved fluorescence, fluorescence polarization, luminescence, electrochemiluminescence and surface plasmon resonance, to name just a few. Development, validation and application of these LBAs requires a thorough understanding of the available methodologies, the analytical requirements of the assay and the regulatory expectations for assay performance based on the intended use of the data.

The various aspects of LBAs in bioanalysis of macromolecules have been the topic of numerous conferences, seminars, workshops and publications. Ligand-Binding Assays provides a comprehensive overview of the field beginning with the history of LBAs, through current technologies and regulatory expectations, concluding with a discussion of future trends. This book contains 13 chapters by 30 authors and covers a range of topics such as development and validation of LBAs for pharmacokinetic assessments, biomarker evaluation, pharmacokinetic/pharmacodynamic analysis and immunogenicity testing, transfer of LBAs in regulated environments, application of automation in LBAs, regulatory compliance and, finally, emerging methodologies in LBAs.

The book begins with the history of LBAs in Chapter 1, starting from radioimmunoassay for insulin, other radiometric and nonradioactive immunoassays, to the formation of the LBAs Bioanalytical Focus Group. It also outlines the

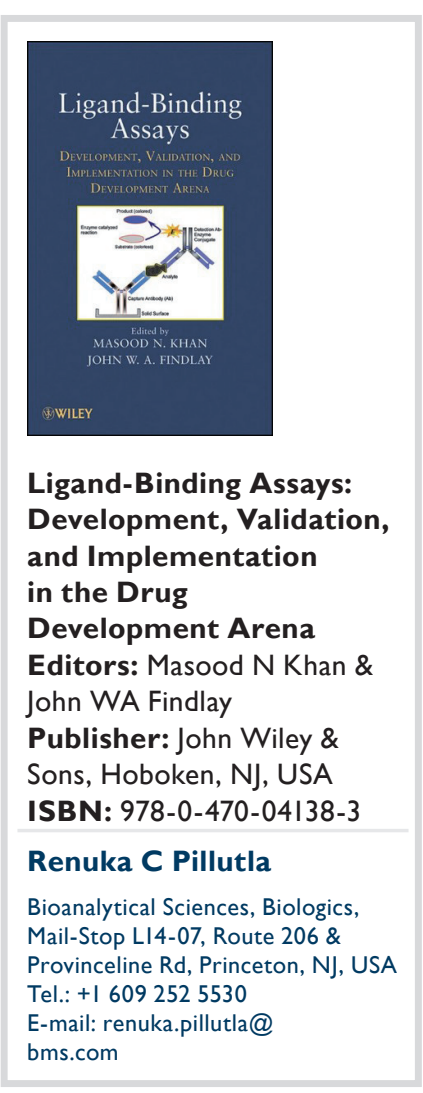


various chapters in the book to provide the reader with a glimpse of what to expect and is helpful in navigating through the book. Chapter 2 presents the use of LBAs in pharmacokinetic studies with specific focus on large molecules. This chapter elucidates in great detail the differences between low-molecular weight molecules versus macromolecules with respect to calibration curves, reference standards, specificity and disposition/clearance. All of these differences and concepts come up several times in later chapters and therefore this detailed introduction serves the reader well as he/she advances to subsequent chapters. Valuable insights into the considerations for LBAs relative to the stage of research or development are presented, as a molecule progresses from discovery, through preclinical and clinical development. This has significant relevance to the bioanalytical lab, as a fit-for-purpose validation of LBAs ensures alignment of the efforts associated with these activities, with the use of the data that is generated.

Chapters 3-5 cover development and validation of LBAs. Challenges and considerations in LBA development are covered in Chapter 3. In particular the practical aspects of assay development such as materials, reagents (coating buffer, blocking solutions, assay diluents, detection labels and substrates), instruments and dataregression software are presented with some case examples as illustrations. This chapter has tremendous value to the bioanalytical scientist at the bench and provides a practical step-by-step guide to assay development and troubleshooting. Chapter 4 outlines the requirements for the validation of LBAs to support good laboratory practice-complaint bioanalysis for pharmacokinetic/ toxicokinetic assessments of biotherapeutics. A step-wise approach to validation from development and prevalidation to prestudy and in-study validation is described. Validation parameters are discussed in great detail. This chapter mirrors recommendations outlined in the US FDA Guidance as well as industry White Papers, providing practical details on the implementation of these recommendations. Chapter 5 discusses statistical aspects of validation such as acceptance criteria, accuracy, precision, linearity, measurement of total error and defining the range of the assay, by presenting ways to understand, estimate and interpret criteria required for assessing the validity of an analytical method.

Other uses of LBAs such as biomarkers, pharmacokinetic/pharmacodynamic analysis and immunogenicity testing are covered in the following three chapters. Chapter 6 focuses on LBAs for biomarkers, with detailed recommendations and examples of the fit-for-purpose approach to biomarker assay development and validation, at the same time highlighting the lack of regulatory guidance. Challenges specific to biomarkers such as obtaining a control matrix free from the endogenous biomarker, specimen integrity, specificity, parallelism and dilutional linearity are presented with some case studies that illustrate these concepts. Chapter 7 draws attention to the use of commercial assay kits for pharmacokinetic/pharmacodynamic analysis, with emphasis on re-optimization, appropriate calibration standards and quality controls, lot-to-lot variability and other issues specific to the measurement of endogenous or related analytes using commercial kits. Chapter 8 describes immunogenicity assays for preclinical and clinical studies. Current regulatory guidances, a risk-based approach to immunogenicity testing, optimization and validation of binding and neutralizing antibody assays, application and interpretation of the data are some of the various aspects discussed in this chapter. This chapter underscores the importance of a sound statistical approach to establishing cut points in the various tiers of immunogenicity testing.

Chapter 9 is dedicated to discussion of reference standards for macromolecules. This concept is first introduced in Chapter 2, discussed in Chapters 3 and 4 and continues to rear its ugly head in subsequent chapters. Chapter 9 takes the bull by its horns and addresses this issue in great detail laying out the challenges with macromolecular reference standards. This chapter describes the history of small molecule reference standards by describing the role of the United States Pharmacopeia (USP) then discusses the features of macromolecular standards which makes it challenging to create USP standards. This chapter also provides guidance on the characterization of non-USP macromolecular reference standards with several case studies for the assessment of purity, potency, concentration and stability of the macromolecule.

A common and nowadays almost integral step in the development of a drug is the outsourcing of the bioanalytical testing to a CRO. This requires identifying and building a relationship with the 'right' CRO. Chapter 10 provides the recipe to building a successful sponsor-CRO relationship, beginning with the evaluation and selection of a CRO, to method transfer, troubleshooting, documenting and auditing. 
With an increase in the number of biologics entering the pipelines of most pharmaceutical and biotechnology companies, there is an increased need for efficiency and throughput without compromising data quality. Chapter 11 provides a step-by-step guide to selecting, integrating and implementing an automated system, highlighting the complexities in the application of automation in LBAs. Compliance considerations including the FDA's 21 CFR Part 11 are also addressed.

Chapter 12 details regulatory expectations for documentation and compliance related to method development, validation, sample analysis and reporting. Emphasis is on the ability to re-construct the study from the documentation to assure data integrity.

Chapter 13 concludes with a discussion of the myriad emerging methodologies based on various technologies such as fluorescence, electrochemiluminescence, surface plasmon resonance, hybridization and chromatography. Future trends and directions for LBAs predict an emphasis on miniaturization of assays for high-throughput drug-discovery support. New technology developments should focus on overcoming limitations of current technologies such as interference from antidrug antibodies on the measurement of circulating drug, and vice versa - interference from circulating drug on the detection of antidrug antibodies.
As the number of biologics in development continues to increase, this book is likely to become a must-have reference for the bioanalytical scientist.

\section{Future perspective}

The shift in the pharmaceutical industry over the past decade, with the scales tipping in favor of biologics, is expected to continue, bringing additional challenges along the way. As some of the early biologics come off patent, biogenerics bring a host of new regulatory considerations to the bioanalytical field. Other developments that will likely have an impact on our approach to bioanalysis of macromolecules are the development of biogenerics, alternate routes of administration and novel drug-delivery systems. Finally, increasing globalization of drug discovery has already led to the initiation of global harmonization efforts across the bioanalytical community.

\section{Financial \& competing interests disclosure}

The author has no relevant affliations or financial involvement with any organization or entity with a financial interest in or financial conflict with the subject matter or materials discussed in the manuscript. This includes employment, consultancies, honoraria, stock ownership or options, expert testimony, grants or patents received or pending, or royalties. No writing assistance was utilized in the production of this manuscript. 